\title{
Invasive Fungal Infection Caused by Exophiala dermatitidis in a Patient After Lung Transplantation: Case Report and Literature Review
}

\author{
Romana Klasinc $\cdot$ Martin Riesenhuber $\cdot$ Andreas Bacher $\cdot$ Birgit Willinger (i)
}

Received: 8 February 2018/ Accepted: 27 May 2018/Published online: 11 June 2018

(C) The Author(s) 2018

\begin{abstract}
This report describes a case of invasive Exophiala dermatitidis infection after double lung transplantation in a 76-year-old man. After thoracotomy, the patient's wound showed dehiscence with purulent secretion. The black yeast was isolated from cultures taken from the wound, and species identification was confirmed by sequence analysis of the internal transcribed spacer (ITS-S2) region. The results of the susceptibility testing showed voriconazole as the most active drug. Despite adaptation of the antifungal therapy the clinical condition worsened, and the patient died. In addition, we evaluated the fungicidal activity of antiseptics towards $E$.
\end{abstract}

Handling Editor: Ferry Hagen.

Romana Klasinc and Martin Riesenhuber have contributed equally to the work.

R. Klasinc $\cdot$ M. Riesenhuber $\cdot$ B. Willinger $(\square)$ Division of Clinical Microbiology, Department of Laboratory Medicine, General Hospital of Vienna, Medical University of Vienna, Vienna, Austria e-mail: birgit.willinger@meduniwien.ac.at

R. Klasinc

Institute for Hygiene and Applied Immunology, Center for Pathophysiology, Infectiology and Immunology, Medical University of Vienna, Vienna, Austria

\section{A. Bacher}

Department of Anaesthesia, Intensive Care Medicine and Pain Medicine, Vienna General Hospital, Medical

University of Vienna, Vienna, Austria dermatitidis and aimed to provide a brief literature review of previously reported infections caused by this rare fungus. To the best of our knowledge, this is the first report of a rapidly progressing invasive fungal infection with $E$. dermatitidis originating from a colonized wound after lung transplantation.

Keywords Exophiala dermatitidis $\cdot$ Lung transplantation $\cdot$ Invasive infection

\section{Introduction}

Exophiala dermatitidis is a saprophytic black yeastlike fungus that rarely infects humans. It is commonly isolated from plant debris and soil and has been detected from indoor-habitats, e.g., dish washers, but also other places with high humidity [1]. E. dermatitidis synthesizes melanin, giving its colonies a dark colour [2]. The respiratory tract of patients with cystic fibrosis (CF) frequently is colonized with E. dermatitidis [3]. The presence of E. dermatitidis triggers inflammatory responses was shown to cause significant airway infections [4] and thus seems to be associated with a more advanced state of disease. Additionally, Exophiala spp. frequently causes soft tissue infections known as phaeohyphomycosis [5], which also was described in case reports after lung transplantation [6, 7]. Infections of the central nervous 
system caused by E. dermatitidis are rare, mostly lethal, and have been described in a population of immunocompetent patients [8]. Furthermore, there are reports of peritonitis caused by $E$. dermatitidis after continuous ambulatory peritoneal dialysis [9].

We herein present - to the best of our knowledgethe first clinical case of an invasive infection with $E$. dermatitidis in a patient presenting with a colonized wound after lung transplantation. Since the diagnosis and therapy of $E$. dermatitidis are very challenging, we aim to draw the attention of clinicians to this fungus in lung-transplant patients.

\section{Case Report}

We report the case of a 76-year-old man who underwent double lung transplantation because of pulmonary fibrosis related to chronic obstructive pulmonary disease. The maintenance immunosuppressive regimen after transplantation included tacrolimus $(0.1 \mathrm{mg} / \mathrm{kg}$ body weight $)$ and prednisolone (15 mg/day, $50 \mathrm{mg} / \mathrm{d}-0.6 \mathrm{mg} / \mathrm{kg}$ on postoperative day followed by tapering). Ten days after surgery, the patient underwent rethoracotomy with left pneumonectomy due to haemorrhagic pleural effusion and subsequent respiratory failure. The antimicrobial prophylaxis after surgery included ciprofloxacin, piperacillin/tazobactam, cefepime, ceftolozane/tazobactam, ceftazidime/avibactam, meropenem, gentamicin, teicoplanin, linezolid and sulfametrole/ trimethoprim which were administered due to colonization with methicillin-resistant Staphylococcus aureus, multidrug resistant Enterobacteriaceae as well as vancomycin resistant Enterococci. In addition, parenteral fluconazole (600 mg/day) and anidulafungin $(100 \mathrm{mg} /$ day) along with liposomal amphotericin B $(10 \mathrm{mg} /$ /every $8 \mathrm{~h})$ administered by inhalation were used for the prophylaxis of fungal infections. In the course of the following days, dehiscence of the thoracotomy wound with exudation of purulent material was noted (Fig. 1). Infection parameters were slightly elevated after transplantation, with a fluctuation range of $1.95-33.03 \mathrm{mg} / \mathrm{dl}$ for C-reactive protein, and $1.67-3.91 \mathrm{ng} / \mathrm{ml}$ for procalcitonin. The patient showed recurrent neutropenia; thus, filgrastim (48 Mio IU/day, s.c.) was regularly substituted. Nineteen culture samples showing growth of $E$. dermatitidis were isolated from the patient's wound after transplantation. The swabs were cultured on Sabouraud (SAB) Gentamicin Chloramphenicol 2 agar (BioMerieux, Marcy l'Etoile, France), incubated for $72 \mathrm{~h}$ at $37{ }^{\circ} \mathrm{C}$ under aerobic conditions, and yielded grey yeast-like colonies. After 3 days, a colour change from grey to black was observed. The isolates were identified by the typical morphological appearance in conjunction with matrix-assisted laser desorption ionization-time of flight mass spectrometry (MALDI-TOF MS) analysis (Bruker Daltonics, Bremen, Germany) with the Bruker Biotyper (version 3.1 software), following the manufacturer's protocol. Since the score value that could be achieved (1.6) was lower than the one defined in the manufacturer's criteria $(\geq 2.00)$ as acceptable for reliable species level identification, sequence analysis of the internal transcribed spacer (ITS-S2) region was performed additionally using the basic local alignment search tool (BLAST) algorithm (https://blast.ncbi.nlm.nih. gov/Blast.cgi) of the National Centre for Biotechnology Information (NCBI). Ninety-nine\% similarity to E. dermatitidis strains ATCC 44504 (GenBank accession no. GU256756) and CBS 207.35 (GenBank accession no. NR121268) was observed, and the same species identification was achieved with the Westerdijk Fungal Biodiversity Institute database (http:// www.westerdijkinstitute.nl, CBS 748.88).

Antimicrobial susceptibility assays for anidulafungin, fluconazole, posaconazole, itraconazole, voriconazole, amphotericin B and isavuconazole were performed using the antifungal broth microdilution (BMD) method described by the European Committee of Antimicrobial Susceptibility Testing (EUCAST) [10], the reference method established by the Clinical Laboratory Standards Institute (CLSI) [11], as well as the E-Test ${ }^{\circledR}$ (BioMerieux, Marcy l'Etoile, France) and MIC Test Strip ${ }^{\circledR}$ (Liofilchem, Roseto 45 degli Abruzzi (Te), Italy). The results are shown in Table 1.

Despite surgical treatment of the wound, the patient's clinical condition deteriorated. Computed tomography scans of the chest showed further dehiscence of the surgical wound with swelling of the soft tissue and marginal storage of the contrast agent as a sign of superinfection on the left side. In addition, the remaining right-sided lung tissue showed small focal coin lesions with pleural effusion. At this stage, cultures from the pleural fluid also revealed growth of E. dermatitidis indicating the development of an invasive fungal infection. The clinical condition did 

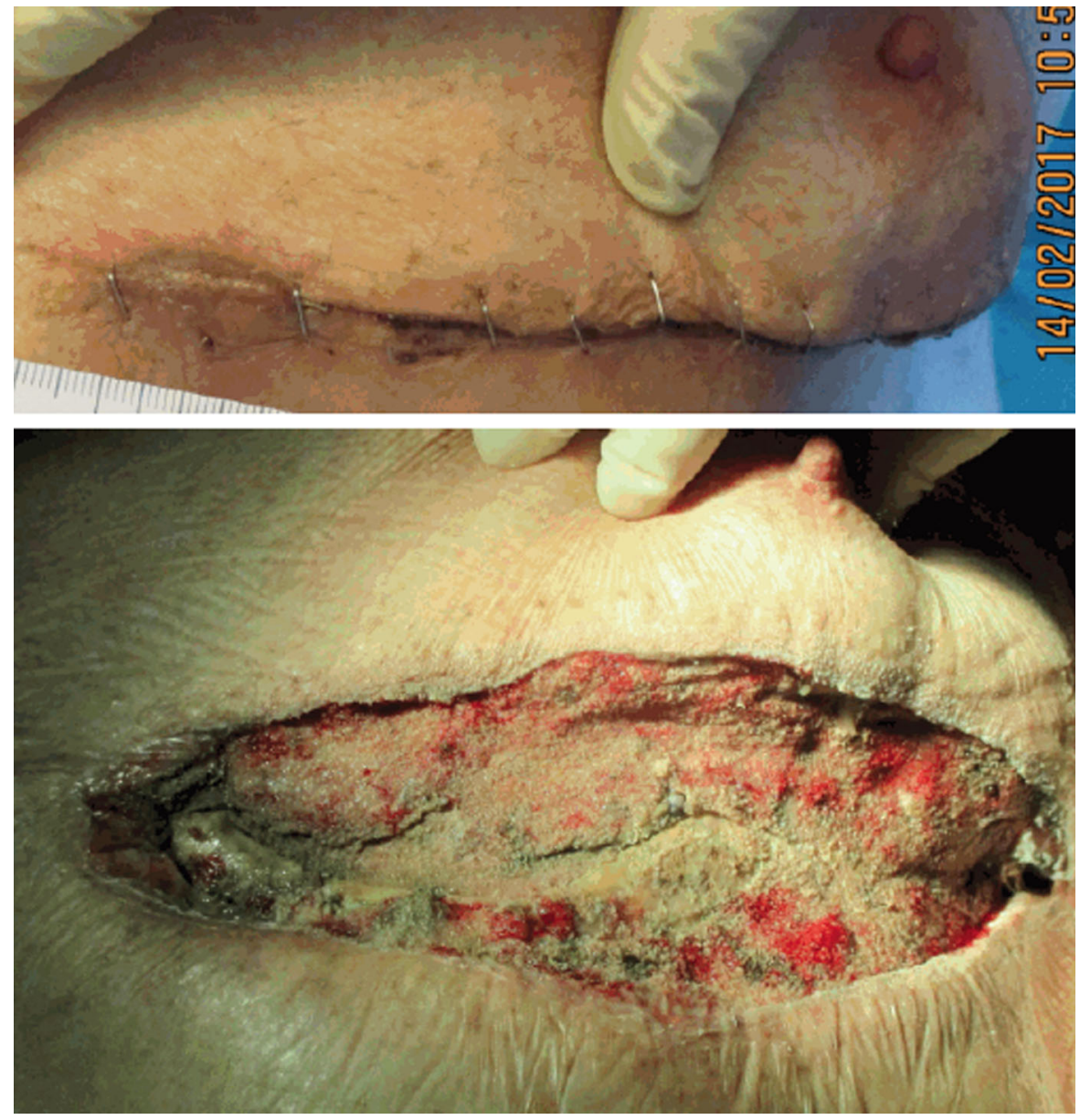

Fig. 1 Progression of the wound in the course of 4 weeks. The upper panel shows purulent material secreted when pressed over the wound margins. The lower panel shows the same wound

not improve even though the therapy was switched to voriconazole ( $300 \mathrm{mg} /$ every $12 \mathrm{~h}$ ), which might be due to the advanced stage of the infection. The patient died 3 days after adapting the antifungal therapy.

To identify the origin of the colonization and possible transmission routes, samples from the patients' environment at the intensive care unitincluding the sink drain and the rubber seal and drain from the dishwasher located in the kitchen as well as sink drains located in the patients' rooms-were taken using sterile liquid transport swab systems $\left(\mathrm{eSwab}^{\mathrm{TM}}\right.$, Hain Lifescience, Nehren, Germany). The swabs were rubbed over the surface of SAB Gentamicin Chloramphenicol 2 agar plates (BioMerieux, Marcy l'Etoile, France) and Sabouraud dextrose broth supplemented with gentamicin, which were then (after 4 weeks) before wound debridement and applying negative-pressure wound therapy

incubated at $37{ }^{\circ} \mathrm{C}$ for up to 3 weeks. None of the samples showed growth of E. dermatitidis.

\section{Quantitative Suspension Test for the Evaluation of Basic Fungicidal Activity of Antiseptics According to EN1275}

C. albicans ATCC 10231 as well as the clinical isolate E. dermatitidis were tested according to EN1275 [12]. The EN1275 Quantitative Suspension Test is designed to evaluate the basic fungicidal activity of chemical disinfectants and antiseptics under various conditions and was applied for the following products: (i) povidone-iodine solution, containing $10 \mathrm{~g}$ povidoneiodine complex in $100 \mathrm{ml}$ water solution yielding 
Table 1 Distribution of MIC range ( $\mu \mathrm{g} / \mathrm{ml})$ determined by E-Test, CLSI and the European Committee of Antimicrobial Susceptibility Testing (EUCAST) broth microdilution (BMD) method for E. dermatitidis

\begin{tabular}{|c|c|c|c|c|c|c|}
\hline & \multicolumn{6}{|c|}{ E. dermatitidis-MIC range } \\
\hline & \multicolumn{3}{|l|}{ CLSI } & \multicolumn{2}{|c|}{ EUCAST } & \multirow{2}{*}{$\begin{array}{l}\text { E-test } \\
24(\mathrm{~h})\end{array}$} \\
\hline & $48(\mathrm{~h})$ & $72(h)$ & $24(\mathrm{~h})$ & 48 (h) & $72(h)$ & \\
\hline Anidulafungin & 8 & 16 & $>16$ & $>16$ & $>16$ & $>32$ \\
\hline Fluconazole & 4 & 8 & 4 & 8 & 8 & 8 \\
\hline Posaconazole & 0.25 & 0.25 & 0.125 & 0.25 & 0.25 & 0.064 \\
\hline Voriconazole & $<0.016$ & 0.032 & 0.032 & 0.064 & 0.064 & 0.023 \\
\hline Isavuconazole & 0.125 & 0.064 & 0.125 & 0.125 & 0.25 & 0.19 \\
\hline Itraconazole & 1 & 1 & 0.5 & 0.5 & 0.5 & 0.125 \\
\hline Amphotericin B & 2 & 2 & 1 & 1 & 2 & 0.38 \\
\hline
\end{tabular}

CLSI Clinical Laboratory Standards Institute

EUCAST European Committee of Antimicrobial Susceptibility Testing

BMD broth microdilution

$11 \%$ available iodine-Betaisodona ${ }^{\circledR}$ (Mundipharma $\mathrm{GmbH}$, Vienna, Austria); (ii) polyhexanide 0.4\%Lavasorb ${ }^{\circledR}$ (Fresenius Kabi AG, Bad Homburg, Germany); (iii) Octenidine dihydrochloride $0.1 \%$ Octenisept $^{\circledR}$ (Schülke \& Mayr GmbH, Norderstedt, Germany), (iv) hydrogen peroxide solution $3 \%$ and alcohol-based disinfectants used in our hospital for hand antisepsis containing Propan-2-ol 45.0 g, Propan-1-ol $30.0 \mathrm{~g}$, Mecetronium etilsulphate $0.2 \mathrm{~g}$ Sterillium ${ }^{\circledR}$ (Paul-Hartmann AG, Heidenheim, Germany). All products were tested at the following concentrations: 80,50 and 20\%; the antiseptics were dissolved in water. The test organisms were diluted in a tryptone sodium chloride solution. An exposure time of $15 \mathrm{~min}$ was considered. Sufficient fungicidal activity can be assumed if an at least $4 \log$ reduction in colony growth is observed after an exposure time no longer than $15 \mathrm{~min}$. According to EN 1275, the recommended incubation time for $C$. albicans ATCC 10231 is $48 \mathrm{~h}$ and is $48 \mathrm{~h}(+24 \mathrm{~h})$ for Aspergillus niger ATCC 16404 at $30{ }^{\circ} \mathrm{C}$ after spreading the suspension on malt extract agar. As there is no recommendation for the testing of other species, for E. dermatitidis a reading was performed every $24 \mathrm{~h}$ up to 3 weeks.

No growth was observed if $E$. dermatitidis and $C$. albicans were exposed to povidone-iodine, polyhexanide, octenidine dihydrochloride and alcohol-based disinfectants. However, even the highest concentration of the hydrogen peroxide solution did not inhibit the growth of E. dermatitidis or C. albicans.

\section{Discussion}

Infections with Exophiala spp. are rare in humans [13-15]. E. dermatitidis, E. xenobiotica and E. oligosperma are the most common species [5]. In the US, these three species are responsible for approximately two-thirds of infections. In vivo tests in immunocompromised mice showed a higher virulence of $E$. dermatitidis compared to E. xenobiotica and $E$. oligosperma [16]. Furthermore, E. dermatitidis is the only neurotropic species and thus can cause infections of the central nervous system (CNS).

In clinical microbiology, the identification of Exophiala is challenging. The use of MALDITOF-a well-established method for the identification of bacteria and yeasts-is limited by the fact that databases used in clinical routine do not necessarily include Exophiala spp. [17]. Our database for example contains only limited spectra for E. dermatitidis; thus, only a low score could be achieved when using MALDI-TOF for the identification of our strain of $E$. dermatitidis. With updated MALDI-TOF databases, MALDI-TOF detection of Exophiala spp. would yield to more reliable results $[18,19]$. 
The gold standard for the identification of $E x$ ophiala spp. is to sequence the internal transcribed spacer (ITS) regions 1 (ITS 1) and 2 (ITS 2) [20, 21]. In our case, sequence analysis of the ITS 2 region confirmed the identification of the isolated strain.

Standardized procedures for susceptibility testing or treatment regimens are not published, although literature suggests that Exophiala spp. usually are susceptible to voriconazole [5, 22], whereas in vitro resistance to echinocandins and fluconazole was observed [23].

According to CLSI and EUCAST, the BMD method is the gold standard for antifungal susceptibility testing for yeasts [10, 11, 24, 25]. However, the less labour intensive E-Test has been demonstrated to be a convenient alternative. Szekely et al. tested 10 isolates of $E$. dermatitidis with both methods and showed a $90 \%$ agreement (within $\pm 2 \log _{2}$ dilutions) of the two methods for itraconazol, while results for amphotericin B only were in agreement in $70 \%$ of the tests [26]. Although the sample size in this study was limited, E-Test seems to be an acceptable alternative, even though results obtained for amphotericin B suggest that the BMD method might provide more reliable results.

We did not observe discrepancies in MIC values obtained by the described methods in our study (Table 1). Furthermore, the MIC values are comparable to those described by others [5, 22, 23, 27, 28].

In patients with localized soft tissue infection caused by $E$. dermatitidis itraconazole in a singlesubstance-regime frequently led to healing [29, 30]. In the literature, only a small number of CNS infections are published. Those generally are associated with a high mortality rate, and a long-course antifungal chemotherapy is necessary $[14,31]$. If involvement of the CNS is suspected, antifungal substances that reach acceptable concentrations in cerebrospinal fluid (CSF)—such as voriconazole—should be selected. A report of the Centres for Disease Control and Prevention described four cases of meningitis caused by $E$. dermatitidis after epidural injections of methylprednisolone and one of these patients was cured with long-term voriconazole-therapy [32]. In murine models, posaconazole showed the best efficacy $[33,34]$.

Pulmonary infections with Exophiala spp. are frequently associated with CF or immunodeficiency. In patients without $\mathrm{CF}$, long-term therapy with itraconazole or voriconazole (up to 7 months) usually is successful $[35,36]$ In patients with $\mathrm{CF}$, Exophiala spp. seem to be under-diagnosed colonizers of the lower respiratory tract [37]. In a prospective study published by Lebecque et al., 5.8\% of CF patients had at least one Exophiala-positive sputum-culture [38]. Further reports on the prevalence of E. dermatitidis in $\mathrm{CF}$ patients suggest a range from $1 \%$ in Germany to around $20 \%$ in Sweden [3, 39]. Possible risk factors identified were age $>12$ years and pancreatic insufficiency [3]. Despite the high prevalence of $E$. dermatitidis in CF patients, and the potential association with disease progression, recommendations in regard to therapy or prophylaxis in those patients are missing. Only a small number of case reports suggested an extended course of voriconazole or itraconazole administration in CF patients with worsening pulmonary symptoms and persistent detection of E. dermatitidis in sputum cultures [40-42].

To date, no data about the antiseptic susceptibility of Exophiala spp. have been published. Therefore, in order to assess the efficacy of antiseptics, we conducted a phase 1 test according to EN1275. Our test results showed inhibition of growth-and thus fungicidal activity, for all tested antiseptics with the exception of hydrogen peroxide, which is still frequently used for wound antisepsis during surgery in our hospital. It is, however, essential to consider that organic matter or blood secreted by wounds might interfere with the fungicidal activity of antiseptics in vivo, and that in vitro activity does not guarantee that the tested antiseptic solutions will be effective under conditions representative for wounds. Shortly after, we performed our experiments, alternative methods like the phase 2/step 2 in vitro test, which is based on EN 14561, have been proposed for this application [43]. Thus, our preliminary results have to be confirmed in a more detailed set of experiments.

In transplant patients, E. dermatitidis mostly causes phaeohyphomycosis usually presenting with nodules containing abscesses or cysts underneath the skin $[44,45]$. These clinical features were also evident in two case reports describing E. jeanselmei phaeohyphomycosis in lung-transplant patients [6, 7]. In contrast to our case, those infections appeared years after lung transplantation and could be cured by surgical excision with or without antifungal therapy. To the best of our knowledge, the case presented herein is the first case describing the presence of the fungus in pleural fluid. 
Evidence concerning transmission routes in the hospital setting is scarce. A nosocomial outbreak of $E$. jeanselmei fungemia related to contaminated deionized water from the pharmacy has been described [46]. In our case, it was not possible to identify the source of infection. We assume that the disregarded colonization with E. dermatitidis was the reason for the development of the invasive fungal infection. However, further research is needed to reveal sources and possible transmission routes of these organisms in the hospital setting.

\section{Conclusion}

In summary, E. dermatitidis colonization can rapidly progress towards an invasive fungal infection. In addition, the detection and identification of this slow growing fungus represent a challenge for the microbiology laboratory, which might lead to delayed-or failed-treatment. Patients at risk, for example, are CF patients colonized by $E$. dermatitidis, as well as immunocompromised patients.

Acknowledgements Open access funding provided by Medical University of Vienna. The authors are especially thankful to Kathrin Spettel for excellent technical assistance. Special thanks are owed to Iris Zeller and Jeremy V. Camp for linguistic advice and revision.

Funding This work was supported by internal funding.

\section{Compliance with Ethical Standards}

Conflicts of interest The authors declare that they have no conflict of interest.

Open Access This article is distributed under the terms of the Creative Commons Attribution 4.0 International License (http:// creativecommons.org/licenses/by/4.0/), which permits unrestricted use, distribution, and reproduction in any medium, provided you give appropriate credit to the original author(s) and the source, provide a link to the Creative Commons license, and indicate if changes were made.

\section{References}

1. Zupančič J, Babič MN, Zalar P, Gunde-Cimerman N. The black yeast Exophiala dermatitidis and other selected opportunistic human fungal pathogens spread from dishwashers to kitchens. PLoS One. 2016;11(2):e0148166.

2. Revankar SG, Sutton DA. Melanized fungi in human disease. Clin Microbiol Rev. 2010;23:884-928.
3. Kondori N, Gilljam M, Lindblad A, Jönsson B, Moore ERB, Wennerås C. High rate of Exophiala dermatitidis recovery in the airways of patients with cystic fibrosis is associated with pancreatic insufficiency. $J$ Clin Microbiol. 2011;49:1004-9.

4. Kondori N, Lindblad A, Welinder-Olsson C, Wennerås C, Gilljam M. Development of $\mathrm{IgG}$ antibodies to Exophiala dermatitidis is associated with inflammatory responses in patients with cystic fibrosis. J Cyst Fibros. 2014;13:391-9.

5. Zeng JS, Sutton DA, Fothergill AW, Rinaldi MG, Harrak MJ, De Hoog GS. Spectrum of clinically relevant Exophiala species in the United States. J Clin Microbiol. 2007;45:3713-20.

6. Chua JD, Gordon SM, Banbury J, Hall GS, Procop GW. Relapsing Exophiala jeanselmei phaeohyphomycosis in a lung-transplant patient. Transpl Infect Dis. 2001;3:235-8.

7. Xu X, Low DW, Palevsky HI, Elenitsas R. Subcutaneous phaeohyphomycotic cysts caused by exophiala jeanselmei in a lung transplant patient. Dermatol Surg. 2001;27:343-6.

8. Kantarcioglu AS, de Hoog GS. Infections of the central nervous system by melanized fungi: a review of cases presented between 1999 and 2004. Stud Mycol. 2004;47:4-13.

9. Greig J, Harkness M, Taylor P, Hashmi C, Liang S, Kwan J. Peritonitis due to the dermatiaceous mold Exophiala dermatitidis complicating continuous ambulatory peritoneal dialysis. Clin Microbiol Infect. 2003;9:713-5.

10. EUCAST. EUCAST E. DEF 7.3.1 EUCAST antifungal MIC method for yeasts. 2017;7.3.1:1-21.

11. Clinical and Laboratory Standards Institute (CLSI). Reference method for broth dilution antifungal susceptibility testing of yeasts, 3rd ed. CLSI document M27-A3. Wayne, PA: Clinical and Laboratory Standards Institute; 2008.

12. European Committee for Standardization. EN 1275. Chemical disinfectants and antiseptics-Quantitative suspension test for the evaluation of basic fungicidal or basic yeasticidal activity of chemical disinfectants and antiseptics-Test method and requirements (phase 1). 2005.

13. Hiruma M, Kawada A, Ohata H, Ohnishi Y, Takahashi H, Yamazaki M, et al. Systemic phaeohyphomycosis caused by Exophiala dermatitidis Systemische Phäohyphomykose verursacht durch Exophiala dermatitidis. Mycoses. 1993;36:1-7.

14. Matsumoto T, Matsuda T, McGinnis MR, Ajello L. Clinical and mycological spectra of Wangiella dermatitidis infections. Mycoses. 1993;36:145-55.

15. Revankar SG, Patterson JE, Sutton DA, Pullen R, Rinaldi MG. Disseminated Phaeohyphomycosis: review of an Emerging Mycosis. Clin Infect Dis. 2002;34:467-76.

16. Calvo E, Rodríguez MM, Mariné M, Mayayo E, Pastor FJ, Guarro J. Comparative virulence of three species of Exophiala in mice. Med Mycol. 2010;48:853-7.

17. Kondori N, Erhard M, Welinder-Olsson C, Groenewald M, Verkley G, Moore ERB. Analyses of black fungi by matrixassisted laser desorption/ionization time-of-flight mass spectrometry (MALDI-TOF MS): species-level identification of clinical isolates of Exophiala dermatitidis. FEMS Microbiol Lett. 2015;362:1-6.

18. Özhak-Baysan B, Öğünç D, Döğen A, Ilkit M, de Hoog GS. MALDI-TOF MS-based identification of black yeasts of the genus Exophiala. Med Mycol. 2015;53:347-52. 
19. Borman AM, Fraser M, Szekely A, Larcombe DE, Johnson EM. Rapid identification of clinically relevant members of the genus exophiala by matrix-assisted laser desorption ionization-time of flight mass spectrometry and description of two novel species, Exophiala campbellii and Exophiala lavatrina. J Clin Microbiol. 2017;55:1162-76.

20. Matos T, Haase G, Gerrits van den Ende AHG, de Hoog GS. Molecular diversity of oligotrophic and neurotropic members of the black yeast genus Exophiala, with accent on $E$. dermatitidis. Antonie Van Leeuwenhoek. 2003;83:293-303.

21. Silva WC, Gonçalves SS, Santos DWCL, Padovan ACB, Bizerra FC. Melo ASA. Species diversity, antifungal susceptibility and phenotypic and genotypic characterisation of Exophiala spp. infecting patients in different medical centres in Brazil. Mycoses. 2017;60:328-37.

22. Sun Y, Liu W, Wan Z, Wang X, Li R. Antifungal activity of antifungal drugs, as well as drug combinations against $E x$ ophiala dermatitidis. Mycopathologia. 2011;171:111-7.

23. Badali H, de Hoog GS, Sudhadham M, Meis JF. Microdilution in vitro antifungal susceptibility of Exophiala dermatitidis, a systemic opportunist. Med Mycol. 2011;49:819-24.

24. Clinical and Laboratory Standards Institute C. Reference method for broth dilution antifungal susceptibility testing of yeasts; Third Informational Supplement-M27-S3. Clin. Lab. Standars Inst.-NCCLS 2008.

25. Lamoth F, Alexander BD. Comparing etest and broth microdilution for antifungal susceptibility testing of the most-relevant pathogenic molds. J Clin Microbiol. 2015;53:3176-81.

26. Szekely A, Johnson EM, Warnock DW. Comparison of E-test and broth microdilution methods for antifungal drug susceptibility testing of molds. J Clin Microbiol. 1999;37:1480-3.

27. Espinel-Ingroff A. In vitro fungicidal activities of voriconazole, itraconazole, and amphotericin B against opportunistic moniliaceous and dematiaceous fungi. J Clin Microbiol. 2001;39:954-8.

28. Fothergill AW, Rinaldi MG, Sutton DA. Antifungal susceptibility testing of Exophiala spp.: a head-to-head comparison of amphotericin $\mathrm{B}$, itraconazole, posaconazole and voriconazole. Med Mycol. 2009;47:41-3.

29. Chen M, Zhang J, Dong Z, Wang F. Cutaneous phaeohyphomycosis caused by Exophiala dermatitidis: a case report and literature review. Indian J Dermatol Venereol Leprol. 2016;82:173-7.

30. Bohelay G, Robert S, Bouges-Michel C, Gerin M, Levy A, Fain O, et al. Subcutaneous phaeohyphomycosis caused by Exophiala spinifera in a European patient with lymphoma: a rare occurrence case report and literature review. Mycoses. 2016;59:691-6.

31. Li DM, De Hoog GS. Cerebral phaeohyphomycosis-a cure at what lengths? Lancet Infect Dis. 2009;9:376-83.

32. Centers for Diasease Control and Prevention. CDC. Exophiala infection from contaminated injectable steroids prepared by a compounding pharmacy-United States, JulyNovember 2002. MMWR. Morb. Mortal. Wkly. Rep. 2002;51:1109-12.

33. Calvo E, Pastor FJ, Guarro J. Antifungal therapies in murine disseminated phaeohyphomycoses caused by Exophiala species. J Antimicrob Chemother. 2010;65:1455-9.

34. Graybill JR, Najvar LK, Johnson E, Bocanegra R, Loebenberg D. Posaconazole therapy of disseminated phaeohyphomycosis in a murine model. Antimicrob Agents Chemother. 2004;48:2288-91.

35. Mukai Y, Nureki SI, Hata M, Shigenaga T, Tokimatsu I, Miyazaki E, et al. Exophiala dermatitidis pneumonia successfully treated with long-term itraconazole therapy. J Infect Chemother. 2014;20:446-9.

36. Bulloch MN. The treatment of pulmonary Wangiella dermatitidis infection with oral voriconazole. J Clin Pharm Ther. 2011;36:433-6.

37. Horré R, Schaal KP, Siekmeier R, Sterzik B, de Hoog GS, Schnitzler N. Isolation of fungi, especially Exophiala dermatitidis, in patients suffering from cystic fibrosis. A prospective study. Respiration. 2004;71:360-6.

38. Lebecque P, Leonard A, Huang D, Reychler G, Boeras A, Leal T, et al. Exophiala (Wangiella) dermatitidis and cystic fibrosis-prevalence and risk factors. Med Mycol. 2010;48:S4-9.

39. Haase G, Skopnik H, Groten T, Kusenbach G, Posselt HG. Long-term fungal cultures from sputum of patients with cystic fibrosis. Mycoses. 1991;34:373-6.

40. Griffard EA, Guajardo JR, Cooperstock MS, Scoville CL. Isolation of Exophiala dermatitidis from pigmented sputum in a cystic fibrosis patient. Pediatr Pulmonol. 2010;45:508-10.

41. Diemert D, Kunimoto D, Sand C, Rennie R. Sputum isolation of Wangiella dermatitidis in patients with cystic fibrosis. Scand J Infect Dis. 2001;33:777-9.

42. Kusenbach G, Skopnik H, Haase G, Friedrichs F, Dohmen H. Exophiala dermatitidis pneumonia in cystic fibrosis. Eur J Pediatr. 1992;151:344-6.

43. Schedler K, Assadian O, Brautferger U, Müller G, Koburger T, Classen S, et al. Proposed phase 2/step 2 in vitro test on basis of EN 14561 for standardised testing of the wound antiseptics PVP-iodine, chlorhexidine digluconate, polihexanide and octenidine dihydrochloride. BMC Infect Dis. 2017; $17: 143$.

44. Lief MH, Caplivski D, Bottone EJ, Lerner S, Vidal C, Huprikar S. Exophiala jeanselmei infection in solid organ transplant recipients: report of two cases and review of the literature. Transpl Infect Dis. 2011;13:73-9.

45. Singh N, Chang FY, Gayowski T, Marino IR. Infections due to dematiaceous fungi in organ transplant recipients: case report and review. Clin Infect Dis. 1997;24:369-74.

46. Nucci M, Akiti T, Barreiros G, Silveira F, Revankar SG, Sutton DA, et al. Nosocomial outbreak of Exophiala jeanselmei fungemia associated with contamination of hospital water. Clin Infect Dis. 2002;34:1475-80. 